\title{
El protagonismo de la familia en la atención temprana de niños y niñas con Síndrome de Down, Chile
}

\author{
Paola Andreucci-Annunziata, Ph.D. \\ Investigadora Universidad Gabriela Mistral, Chile * \\ Camila Morales-Cabello, Lic. \\ Investigadora Universidad Gabriela Mistral, Chile **
}

paola.andreucci@ugm.cl

\section{Resumen (descriptivo)}

Se presenta un modelo de intervención en atención temprana en niños y niñas con SD, inscribiéndose en el enfoque de derechos y la perspectiva de la educación inclusiva en primera infancia. Para abordar las necesidades educativo-sanitarias especiales de estos niños y niñas, de entre 0 y 4 años, se diseña un programa de intervención asociado al grado de discapacidad cognitiva-afectiva-relacional previamente diagnosticado. El seguimiento se desarrolla en un taller de acompañamiento con progenitores y en atenciones con los niños y niñas en un centro universitario de atención temprana. Este programa entrega a la familia herramientas para constituirse en agentes activos de cuidado en el proceso de desarrollo y aprendizaje de sus hijos e hijas. Se evidencian sus resultados y las propuestas de mejora que provienen de progenitores y cuidadores.

\section{Palabras clave}

Atención temprana, Síndrome de Down, enfoque de derechos, discapacidad, familia.

\section{Palabras clave autoras}

Educación inclusiva.

\section{Thesauro}

Tesauro de Ciencias Sociales de la Unesco, Iresie.
Para citar este artículo

Andreucci-Annunziata, P., \& MoralesCabello, C. (2019). El protagonismo de la familia en la atención temprana de niños y niñas con Síndrome de Down, Chile. Revista Latinoamericana de Ciencias Sociales, Niñez y Juventud, 17(2), 1-21. doi:10.11600/1692715x.17207

Historial

Recibido: 14.06 .2017

Aceptado: 20.10.2017

Publicado: 30.06 .2019

Información artículo

Este artículo se enmarca en el estudio «Evaluación de la atención temprana de un grupo de niños y niñas con Síndrome de Down y sus familias por parte de las áreas de Educación y Salud de la Universidad UCINF» (Proyecto acogido al Art. ${ }^{\circ}$ 69 de la Ley 18681) implementado entre el 01 de enero de 2016 y el 31 de diciembre de 2016, con apoyo del Ministerio de Educación de Chile. Área: Educación (Capacitación, Pedagogía). Subárea: Educación Especial. 


\section{The role of the family in the early care of children with Down's Syndrome, Chile}

Abstract (descriptive)

An intervention model for Early Childhood Assistance (ECA) is presented for children with Down's Syndrome. This is based on a human rights approach and an inclusive early childhood education perspective. To address the special educational and health needs of children with Down's Syndrome between the ages of 1 and 4 years, an intervention program based on the previously diagnosed degree of cognitive-affective-relational disability was designed. The follow-up session is carried out during a workshop with parents and in a practical session with the children at an ECA university center. This program gives families tools to become active agents in the process of their children's learning and development. The results of this work and the improvement proposals developed by the parents and caregivers themselves are described in the article.

\section{Keywords}

Early attention, Down's syndrome, rights approach, disability, family.

Authors' keywords

Inclusive education.

\section{O papel da família no cuidado precoce de crianças com síndrome de Down, Chile}

\section{Resumo (descritivo)}

Apresenta-se um modelo de intervenção em Atenção Precoce em crianças com síndrome de Down, registrado na abordagem dos direitos humanos e perspectiva da educação inclusiva na primeira infância. Para atender às necessidades educacionais e de saúde especiais destas crianças, com idades entre 1 e 4 anos, um programa de intervenção associada com o grau de deficiência relacional-afetivo-cognitiva é desenhado previamente e diagnosticado. $\bigcirc$ monitoramento ocorre em uma oficina de acompanhamento com os pais e os cuidados com as crianças em uma universidade atenção precoce. Este programa fornece ferramentas familiares para se tornarem agentes ativos no processo de desenvolvimento e aprendizagem dos seus filhos. Resultados e propostas de melhorias que vêm dos mesmos pais e cuidadores são evidentes.

\section{Palavras-chave}

Atenção precoce; síndrome de Down; enfoque de direitos; deficiencia; família.

\section{Palavras-chave autores}

Educação inclusiva.

\section{Información autoras}

[*] Psicóloga y Licenciada en Psicología por la Universidad de Chile. Magíster en Ciencias de la Educación por la Pontificia Universidad Católica de Chile. Doctora en Ciencias de la Educación por la Pontificia Universidad Católica de Chile. Académica e Investigadora de la Pontificia Universidad Católica de Chile. Directora Centro de Investigación en Educación Universidad Gabriela Mistral (CIE-UGM).Directora Centro de Estimulación Temprana Universidad Gabriela Mistral (CET-UGM). Orcid: 0000-0002-4438-0119. Índice H5: 4. Correo electrónico: paola.andreucci@ugm.cl

[**] Socióloga y Licenciada en Sociología por la Universidad Academia de Humanismo Cristiano. Se encuentra realizando sus estudios de Maestría en Educación en la Universidad Nacional de La Plata. Orcid: 0000-0003-0217-972. Índice H5: 1. Correo electrónico: camila.morales@ugm.cl 


\section{Introducción}

La presente propuesta aborda la dimensión del trabajo con familias que tienen uno o más integrantes, niños o niñas entre o y 4 años, con Síndrome de Down (SD), en el marco de un proyecto formativo-interventivo en atención temprana (AT) desarrollado al interior de una institución universitaria privada chilena. La relevancia de la temática se presenta desde la literatura científica reciente que ratifica que el apoyo de la familia es fundamental cuando se aborda la condición de discapacidad (Guralnick, 2016; McWilliam, 2016; Simón, Giné, \& Echeita, 2016). Asimismo, desde el enfoque de derechos humanos para la discapacidad, que se expresa tanto en el acceso como en la habilitación necesaria y pertinente de los servicios sociales, cuidados sanitarios y educativos para todos y todas sin excepción; este cuestiona los modelos médicos focalizados en el déficit, la falta o la carencia como limitantes irremediables centradas exclusivamente en las personas en condición de discapacidad más que en las limitantes que provienen desde la misma sociedad y sus marcos regulatorios homogenizantes (Booth \& Ainscow, 2015; Rodríguez, Jiménez, \& Sánchez, 2018). Se asume la Convención de los derechos del niño (Lozano-Vicente, 2016), entendida esta como una normativa regulatoria que protege los derechos de niños, niñas y adolescentes, donde no solamente se explicitan los derechos con los que cuentan estos individuos, sino también las obligaciones del Estado y la familia de proveer orientación a los padres y madres y, desde ellos, a sus hijos(as) para un claro ejercicio de sus derechos conforme a la evolución y potenciación de sus capacidades (Morales-Cabello, Andreucci-Annunziata, \& Eisendecher, 2018; Palacios \& Bariff1, 2014). Lo anterior se plasma, entre otras aristas fundamentales del enfoque de derechos (puesto que son reconocidas como personas sujetas de derechos, los principios y orientaciones de una educación inclusiva efectiva en la primera infancia), en contextos familiares, no familiares, inéditos o inhabituales (Cara, Sola, Aznar, \& Fernández, 2015; Palomares, Sánchez, \& Garrote, 2016).

Según las teorías sistémicas familiares, por su parte, lo que afecta a cualquier integrante de la familia con algún grado de discapacidad inevitablemente va a repercutir en 
el resto de sus miembros. Por consiguiente, tanto el foco en el niño o niña como en la familia son ambos igualmente importantes debido a que, si desde la familia existe una transformación positiva en vías del crecimiento, esta va a repercutir satisfactoriamente en el bienestar y desarrollo del niño o niña, y viceversa (Moreno \& Granada, 2014). Entre más centrado el trabajo en las familias a través de la capacitación, empoderamiento, atribución de autoridad y autogestión de sí mismas, más van a poder desarrollar sus fortalezas y recursos en las prácticas de crianza y en los cuidados cotidianos que todos sus integrantes requieren (Viloria \& González, 2017). Esto significa que la familia se constituye en un eje fundamental y motor de cambio para cada uno de sus miembros, especialmente para aquellos eventualmente más vulnerables por cursar etapas tempranas de su desarrollo o por transitar con algún grado de discapacidad (Simón et al., 2016). Este enfoque sistémico también es aplicable a los equipos y duplas profesionales de trabajo, quienes se apoyan y retroalimentan en sesión y fuera de ella, construyendo saberes conceptuales, procedimentales y actitudinales conjuntos desde el ámbito de la educación y de la salud (Andreucci-Annunziata, 2016). Asimismo, los padres y madres contribuyen en sesión, siempre presentes por cierto, no solo como fuentes de información sino también en la elaboración de hipótesis conjuntas sobre el desarrollo o potencial estancamiento del mismo en sus hijos e hijas, en el diseño de estrategias de afrontamiento y en la evaluación del proceso y de los resultados de su implementación.

Algunos autores plantean que los padres y madres con un hijo o hija con SD, en primera instancia, buscan ayuda en los centros de estimulación o de atención temprana (Esquivel, 2015; Shulman, 2016); no obstante, el trabajo se ve fortalecido una vez que logran crear una red de cuidados (Ancheta, 2018; Moreno \& Granada, 2014), con padres y madres que tienen un hijo o hija con el mismo síndrome y se dan cuenta que los sentimientos y dudas que afloran son compartidos según cada etapa de desarrollo (Guralnick, 2016). Si esta instancia es, además, apropiadamente guiada por profesionales capacitados que intervienen, educan o psicoeducan y se transforman en mediadores del desarrollo familiar, el proceso se torna de llevadero a fructífero y el aprendizaje más significativo y profundo para todos los agentes participantes en el dispositivo de AT.

\section{Objetivos}

Para este reporte de caso (Stake, 2010) se seleccionaron dos objetivos del modelo de intervención general propuesto: 
1) Contribuir al bienestar subjetivo y al protagonismo de madres, padres o cuidadores principales de niños y niñas con condición de SD que asisten a un centro de AT, en su rol de cuidadores.

2) Afianzar el trabajo sistémico colaborativo de duplas profesionales a través de las visitas domiciliarias y el trabajo conjunto con los padres o madres en los boxes de atención en un centro de estimulación y atención temprana urbano.

\section{Marco Conceptual}

Este marco de referencia aborda las nociones de AT realizando una breve revisión de la evolución conceptual y procedimental desde la instalación de la estimulación precoz hasta la intervención temprana multidisciplinaria que se propugna hoy en día, y los enfoques centrados en el rol de la familia, paraguas paradigmático en el cual se ampara la presente propuesta.

\section{Atención temprana: conceptualización e implicancias}

La AT se ha concebido como un pilar para el logro de los procesos de aprendizaje y la integración de niños, niñas y jóvenes con distintos tipos y grados de discapacidad en la educación y en la vida activa de sus sociedades de pertenencia o referencia (Guralnick, 2016). Se entiende por atención temprana al

conjunto de intervenciones dirigidas a la población infantil de o-6 años, a la familia y al entorno, que tienen por objetivo dar respuesta lo más pronto posible a las necesidades transitorias o permanentes que presentan los niños con trastornos en su desarrollo o que tienen el riesgo de padecerlos. Estas intervenciones, que deben considerar la globalidad del niño, han de ser planificadas por un equipo de profesionales de orientación interdisciplinar. (Federación Estatal de Asociaciones de Profesionales de Atención Temprana, 200o, p. 10)

En el vasto espectro de las discapacidades de las que se ocupa o debiera ocuparse el dispositivo de AT y sus equipos multidisciplinarios — salud, educación, servicio social— se encuentran las discapacidades mentales, intelectuales o cognitivas y, especificamente, el SD. El Libro blanco de la atención temprana (Federación Estatal de Asociaciones de Profesionales de Atención Temprana, 200o) planea como objetivos para estos niños y niñas: 1) reducir al máximo los efectos de una deficiencia sobre el desarrollo global del niño o de la niña; 2) considerar al niño y a la niña como sujetos activos de la intervención; 
3) adaptar el entorno a las necesidades específicas del niño o la niña; 4) atender las necesidades de la familia que derivan de la situación de tener que atender, a su vez, a las necesidades médicas y educativas de un niño o niña con discapacidad. A partir de estos objetivos se ha planteado en Iberoamérica y el mundo un trabajo de educación inclusiva en primera infancia (aún en curso), con foco en adelantar la edad al diagnóstico y a la remisión a valoración en AT para favorecer un sistema educativo que considere las variables del niño o de la niña y su entorno. Por otra parte, los sistemas sanitarios y de servicio social debieran determinar el lugar de residencia y reducir los tiempos de espera hasta la valoración por un equipo multidisciplinar específico, reduciendo, a su vez, los costos económicos, sociales y emocionales asociados para la familia. Mientras esto ocurre, las familias se transforman en los grandes y a veces únicos soportes para la conducción de su hijos e hijas por procesos formativos y de intervención que, guiándose por pautas iniciales adquiridas en algunos centros sanitarios, intentan replicar en sus hogares sin dejar de lado su insustituible labor parental en los cuidados cotidianos y hábitos de la vida diaria.

Desde hace varias décadas se ha sabido que la intervención multidisciplinaria en AT y la implicación de profesionales y familia en el abordaje precoz de los niños y niñas en situación de discapacidad y la necesidad de educación inclusiva, cambia el pronóstico y las perspectivas de desarrollo de estas poblaciones, incluso en los casos de afectaciones anatómicas muy severas (Guralnick, 2016). No obstante, esta aproximación no ha ocupado un lugar relevante sino desde muy entrado el siglo XX. Haciendo un poco de historia, entre la década de los setentas y noventas se puso especial atención al niño y niña desde la perspectiva de la estimulación precoz a cargo de un equipo especializado; lo anterior, desatendiendo, hasta cierto punto, el contexto y las condicionantes medioambientales del hogar y de la escuela, en los casos de niños y niñas que ya estaban en edad y condiciones de acceder al jardín de infantes. Aún en esta etapa inicial, los programas de estimulación precoz de mayor éxito resultan ser en los que intervienen los padres y madres, independientemente de la modalidad sensorial empleada. Sin embargo, estos programas tienden a fallar en mayor o menor medida o no llevarse a cabo plenamente por la falta de recursos económicos de las familias, a pesar de contar con cierto amparo en la legalidad vigente (Guralnick, 2011). No obstante, los avances en las neurociencias al iniciarse el s. XXI, han demostrado el valor de las experiencias tempranas en la organización cerebral y cómo estas experiencias al aumentar el conocimiento del tiempo perinatal, ubican a los padres y madres como cuidadores primarios fundamentales (Andreucci-Annunziata, 2016). 


\section{Enfoques centrados en la familia}

En las dos últimas décadas, en esta materia se consolida el estudio y la investigación de la infancia temprana (o-4 años), produciéndose avances teóricos y conceptuales con el desarrollo de un marco común de referencia; además, se logra apoyo científico sobre la efectividad de los programas y se obtienen consensos en los principios marco para el diseño e implementación de sistemas de AT. Junto a ello, se produce un gran y renovado impacto en la generación de políticas públicas en variados países del mundo, desarrollándose diversos tipos de programas de intervención infantil; prácticas estas basadas en evidencia científica y en experiencias comparadas, diseño e implementación de sistemas de atención temprana con focos formativos, interventivos y sociales cuya efectividad se logra evaluar sistemáticamente (Guralnick, 2016). Los autores son bastante claros en señalar la importancia de la familia (padres y madres) en su rol de cuidadores y formadores durante todo este proceso (García, Escorcia, Sánchez, Orcajada, \& Hernández, 2014).

Este giro de la AT hacia el protagonismo de las familias responde a lo que se empieza a conocer como «enfoques centrados en la familia» (Álvarez, Alonso, \& Amor, 2016; McWilliam, 2016) y que tienen como propósito la consideración de los ámbitos naturales de inserción y tránsito del niño o niña; es decir, del contexto hogar-escuela (Simón et al., 2016) en el abordaje de la discapacidad y la inclusión de madres, padres o cuidadores con sus respectivas prácticas de crianza como agentes fundamentales en los procesos de desarrollo y aprendizaje de sus hijos e hijas impactando, a su vez, en la propia organización familiar en tanto estructura, dinámica, roles y sistemas de apego y cuidado (Flores \& Porges, 2017; González, Blanco, \& Herrero, 2017; Morales-Cabello et al., 2018; PalaciosHernández, 2016; Viloria \& González, 2017). Las funciones, posiciones y roles que pueden desempeñar los integrantes de la familia, especialmente (aunque no exclusivamente madres y padres, requieren acompañarse desde el equipo multiprofesional e interdisciplinar teniendo en cuenta los principios siguientes que se resumen en la figura 1.

La confianza y el respeto depositados en las familias resume gran parte de los principios de este nuevo enfoque que ya se delinea en el Libro blanco de la atención temprana, pero que no se ha implementado con toda la fuerza que se requiere para apoyar y sostener los cambios impulsados por el equipo multiprofesional y multidisciplinario, a lo largo del tiempo. Esta labor, no obstante, requiere de acompañamiento para estos padres y madres y una genuina valoración de sus posturas, planteamientos, sentimientos, actitudes y concepciones frente a sus hijos/as y frente a sí mismos/as. 


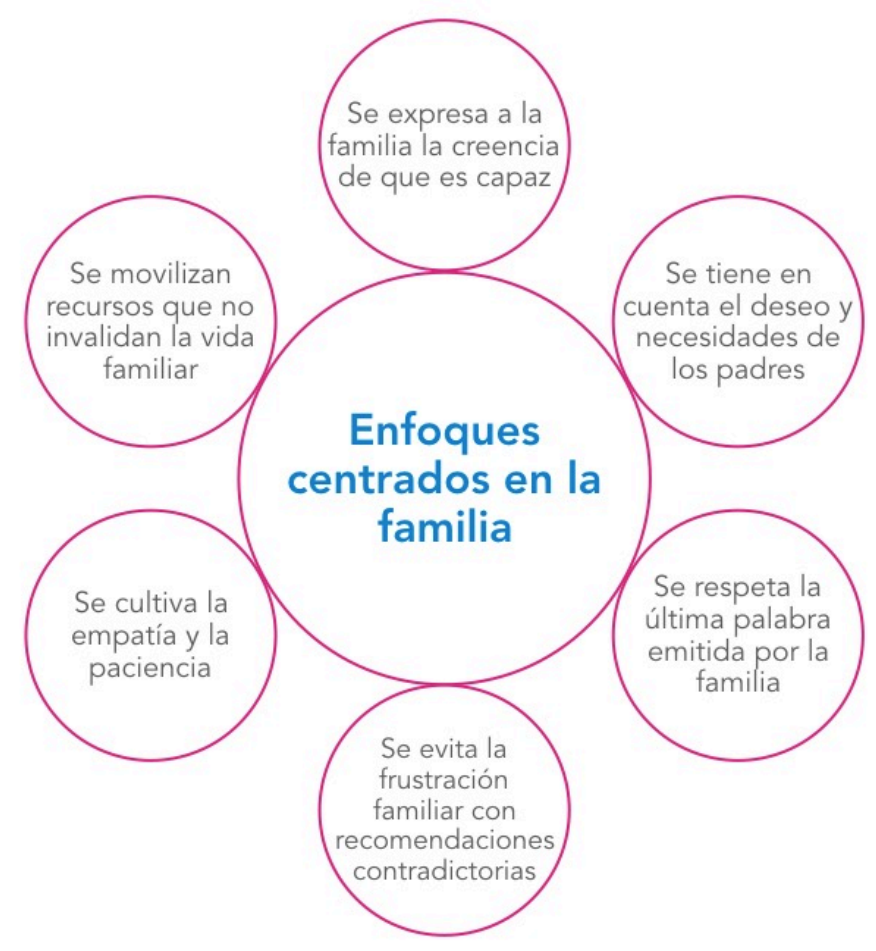

Figura 1. Principios de los Enfoques Centrados en la Familia

\section{Método de intervención}

El presente reporte de caso (Stake, 2010) se inserta en un modelo de intervención cuyo método contempla varias fases, sucesivas o recursivas, dentro del marco de los enfoques centrados tanto en la familia como en los derechos de niños y niñas, que pueden nominarse como inclusivos y describirse sintéticamente anudadas a una determinada modalidad de trabajo multi e interdisciplinar.

El estudio es de carácter descriptivo y utiliza una estrategia mixta de indagación. Tiene como población objeto a 25 niños y niñas con SD, quienes asisten al centro AT, sus familias y equipos de educadoras de los jardines infantiles.

El enfoque cuantitativo de investigación supone la construcción de encuestas que fueron aplicadas a las madres, padres o cuidadores y, paralelamente, al equipo educativo de los jardines a través de una modalidad electrónica (en dos momentos: inicial y final). La invitación se envió mediante correos electrónicos obtenidos de las bases de datos generadas por el equipo de investigación del centro AT. El seguimiento se realizó a través de este mismo procedimiento. Por otro lado, se aplicó la Escala de parentalidad positiva 
e2p, instrumento cuyo objetivo es identificar las competencias parentales, formativas, de orientación normativa y de cuidado, que los/as adultos/as responsables utilizan al relacionarse con sus hijos e hijas. El análisis de la información se realizó de forma estadística para el cuestionario: 1) caracterizando a los/las entrevistados/as; 2) determinando a través de la selección de un grupo de variables, si tuvo o no impacto el modelo de intervención; 3) identificando los factores que han incidido en dicho impacto; y 4) comparando los resultados según género, quintil de ingresos, comuna de procedencia y nivel educacional. La presentación inicial de estos resultados ha sido objeto de una comunicación previa (Morales-Cabello et al., 2018).

Ahora bien, la estrategia cualitativa tuvo por objetivo profundizar en aquellos hallazgos que se realizaron a partir del análisis de los datos, principalmente en lo referido a expectativas, evaluación de los procesos de intervención o apoyo, diseño e implementación de cuidados y percepción de su rol como madres, padres o cuidadores/as. Para el presente reporte se seleccionaron y analizaron los registros de 20 sujetos correspondientes a 10 familias (10 padres y 10 madres), de recursos bajos y moderados, quienes accedieron, mediante formulario de consentimiento informado, a las entrevistas en profundidad iniciales; estos completaron su participación en el grupo focal de cierre al finalizar 11 sesiones de intervención. Para el procesamiento de los datos se utilizó el programa Atlas.ti (v. 7.6), estableciendo las categorías que se desprendieron del marco teórico y las emergentes, con la perspectiva del análisis de contenido.

\section{Fases de intervención en AT}

1) Visitas domiciliarias: el trabajo se inicia con la visita, bajo consentimiento informado de las familias, en sus respectivos hogares. Estas familias, que han solicitado atención a través de la Red de Atención Temprana (Chile) desde sus centros sanitarios de atención primaria o por sugerencias de madres y padres o simplemente por motivación personal, son contactadas desde el centro y registradas las horas de visita con la dupla profesional seleccionada para este fin. La visita consiste en una entrevista con los padres y demás familiares presentes en el hogar, la realización de una anamnesis y el registro de un protocolo de observación de contexto infraestructural y socioeconómico con el propósito de ajustar las indicaciones de estimulación con la niña o niño a la realidad familiar y de residencia respectivas.

2) Atención en duplas profesionales: la dupla profesional, compuesta por un especialista del ámbito educativo y un especialista del ámbito de la salud, diseña un protocolo de 
atención para el niño o niña que ha sido visitado con atenciones semanales o quincenales en el centro. Este protocolo se inicia con la aplicación del Test de aprendizaje y desarrollo Infantil (Tadi) validado en población preescolar chilena (Pardo, Gómez, \& Edwards, 2012) y continúa con las pautas de estimulación que la dupla profesional defina para cada caso. Los referentes de estimulación se sitúan en los enfoques de juego libre (Rockel \& Sansom, 2015) y de enriquecimiento instrumental de Feuerstein (Kosubik, Síthova, Kajanová, \& Rác, 2018), adicionalmente a los propios de cada especialidad o disciplina comprendida en la dupla. Desde el punto de vista educativo se consideran las bases curriculares de la educación parvularia y desde el punto de vista sanitario, las propuestas específicas provenientes de la fonoaudiología, kinesiología, terapia ocupacional y psicología.

3) Taller de habilidades sociales: en función del diagnóstico previo, algunos niños y niñas son incorporados a talleres de habilidades sociales cuyo propósito es desarrollar habilidades cotidianas y relacionales con sus pares en un ambiente facilitador mediado por una educadora de párvulos o educadora diferencial, según sea el caso. Estos talleres que se extienden por 12 sesiones, se conciben de naturaleza abierta (con ingreso y egreso de niños(as) a lo largo del tratamiento de cada uno/a de ellos/as) y de tipo complementario a la intervención en duplas profesionales desarrollada a lo largo del año con la periodicidad y foco antes descritos.

4) Talleres temáticos: por requerimiento de las familias o por acuerdo del equipo de trabajo en las reuniones de equipo semanales, se programan talleres sobre temas que ha resultado recurrentes en la sesiones de duplas, en las entrevistas con los padres o en las visitas domiciliarias. Se han abordado los siguientes: psicomotricidad, lenguaje, juego, cuidados cotidianos, control de esfínteres y alimentación con sondas. El formato de tipo educativo se matiza con dinámicas interactivas y las interrogantes-ejemplificaciones de los propios padres y familiares (hermanos/as, fundamentalmente).

5) Programa para padres, madres y cuidadores: en modalidad de talleres semanales, a desarrollarse los días sábados, el programa tiene el propósito de contribuir al bienestar subjetivo de madres, padres o cuidadores principales de niños y niñas con condición de SD. Para ello, evalúa competencias parentales presentes en los participantes del taller para contribuir, finalmente, al bienestar subjetivo en sus dimensiones cognitiva y afectiva en un contexto relacional de trabajo interfamilias. El facilitador - psicólogo en este caso-gatilla las interacciones en función de la comprensión diagnóstica previa realizada, para luego mediar las reacciones emocionales a lo largo del taller en íntima conexión 
con las etapas de desarrollo y reacción al tratamiento implementado por el centro, en sus hijos e hijas.

6) Articulación con jardines infantiles: en los casos de los niños y niñas que se encuentran en etapa de transición al sistema escolar, se favorece este tránsito realizando los contactos con los jardines infantiles que los recibirán o ya los han incluido. Este contacto consiste en coordinación de visitas a los establecimientos preescolares, reunión con las educadoras de párvulos e invitación de las mismas a los talleres temáticos; lo anterior con el propósito de compartir conocimiento actualizado sobre ámbitos relevantes para el trabajo con los niños y niñas e intercambio con los padres y madres que participan de los mismos talleres. Ello facilita la construcción de un conocimiento común y la generación de las confianzas necesarias para avanzar en los aprendizajes e intervenciones propuestas.

En síntesis, todas las fases o ámbitos del modelo propuesto respetan los derechos de los niños, niñas y familias avanzando al ritmo de intervención que unos y otras imponen al trabajo conjunto con los y las profesionales del equipo del centro AT. Estos últimos disponen su saber, compromiso profesional, personal y sus respectivas experticias a la individualidad de cada caso, de acuerdo al protocolo de intervención diseñado ad-hoc.

Para este reporte de caso (Stake, 2010) se seleccionaron las dos fases o ámbitos de actividades que se conciben como más innovadoras, sistémicamente valiosas y consistentes con un enfoque centrado en la familia y que, consecuentemente, han resultado especialmente valoradas por los/as usuarios/as del centro, al imprimirles mayor protagonismo: el trabajo conjunto desarrollado por las duplas profesionales con los niños y niñas con SD y el programa de acompañamiento para padres, madres o cuidadores/as de niños o niñas con SD.

\section{Trabajo en duplas profesionales}

El trabajo de las duplas profesionales contempla sesiones de intervención con ambos especialistas presentes. Los profesionales intervienen en las sesiones con periodicidad semanal o quincenal, previo diagnóstico de cada niño o niña y su familia realizado en la respectiva visita domiciliaria; de modo conjunto preparan las sesiones de intervención y realizan la retroalimentación o sugerencias a los familiares con sus informes de progreso. El número de familias que abordan las duplas profesionales asciende a 50, encargándose cada dupla directamente de 10 de ellas. Estas duplas son responsables de gestionar el caso asignado y coordinan, registran, monitorean y evalúan los avances de cada familia, sugiriendo derivaciones intraequipo, traspaso a otras duplas con mayor grado de exper- 
ticia en el ámbito relevante-emergente del caso asignado (por ejemplo, emergencia de la separación de los padres que amerita derivación a dupla psicosocial) o derivación a la Red AT Chile de acuerdo a zona de residencia, grado de consolidación de los equipos y solicitud de los padres y las madres.

\section{Programa de intervención con padres, madres o cuidadores}

El programa, en modalidad de taller interactivo cuyo formato es el atribuido a los grupos triangulares (Andreucci-Annunziata \& Guajardo, 2016), contempla once sesiones en total, una vez a la semana, de hora y media de duración en promedio, de preferencia día sábado por la mañana. Los padres ingresan por voluntad propia o a sugerencia de las duplas profesionales que han realizado la visita domiciliaria y el seguimiento del caso. La primera sesión se destina a la presentación del programa, de los encargados de implementarlo (dupla de psicólogos hombre-mujer), de los y las participantes y a la detección de necesidades en torno a las cuales los y las asistentes desean trabajar. Las sesiones siguientes se realizan en dos momentos: el primero implica la psicoeducación en relación a la estimulación y atención tempranas, desde diversas temáticas relativas a los cuidados cotidianos (higiene personal, rutinas de vestimenta, control de esfínteres, entre las más relevantes). Un segundo momento, relacionado con el trabajo en habilidades parentales (educativas, de orientación normativa y de protección afectiva), desde el reconocimiento de las mismas hasta el entrenamiento en ellas. En este momento se hace efectiva la técnica de grupos triangulares ya que ambos facilitadores se ubican en el lugar simbólico de la acogida-orientación, con una grupalidad orientada hacia una experiencia de realidad que favorece la creatividad, a la vez, que se distinguen e identifican las fases del proceso grupal (desde la dependencia del criterio experto a la independencia y autogestión) y los niveles de creación lingüística en el dialogo grupal (Andreucci-Annunziata \& Guajardo, 2016). La finalización, en la sesión número once, se realiza con la aplicación de la encuesta de satisfacción, la implementación de un grupo focal y un cierre. Adicionalmente, los padres que participaron en este reporte, organizaron una convivencia al aire libre como retribución al equipo profesional y al trabajo conjunto realizado.

\section{Modalidad de intervención en AT}

Las fases previamente descritas se insertan en una modalidad y ética de trabajo colaborativo que considera el aporte de cada cual en la construcción del dispositivo de AT. Ello se plasma en una labor reflexiva e interdisciplinaria de los equipos de trabajo que 
puede graficarse como una construcción conjunta de ladrillos ubicados sutilmente en la posición correcta del dispositivo (figura 2).

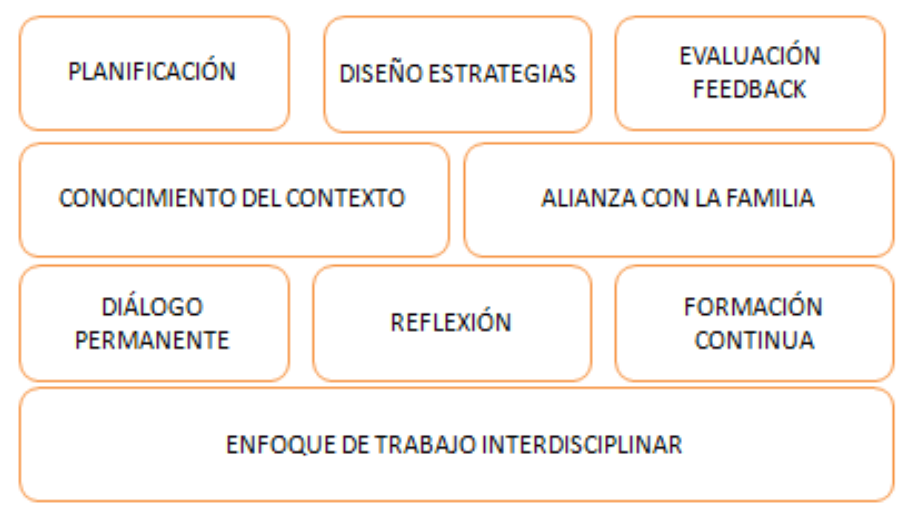

Figura 2. Modalidad de trabajo colaborativo.

Tanto el diálogo permanente como la reflexión y formación continua del equipo profesional favorece el trabajo interdisciplinario y el debido respeto y consideración a la perspectiva del otro. La familia aporta el conocimiento del contexto que solo se logra con una apropiada y genuina alianza de trabajo cuya planificación y diseño conjunto de estrategias favorece la evaluación del proceso y de los resultados de cada fase del modelo propuesto. La obtención de información y generación de confianzas a través del diálogo informal, de las entrevistas grupales y de las encuestas formales, retroalimenta el modelo y aporta a optimizar la propuesta en pro del desarrollo vital y del aprendizaje de niños, niñas y sus respectivas familias de pertenencia extendiéndose, incluso, hacia sus grupos de referencia (agrupaciones de padres, centros recreativos comunales).

\section{Resultados}

Las evidencias se presentan a partir de la sistematización de los resultados del grupo focal en modalidad de entrevistas grupales (Stake, 2010). Como ya se ha señalado, participaron 10 padres y 10 madres de un total de 25 progenitores que iniciaron el proceso de AT, durante el período 2016. El grupo focal se desarrolló en diciembre de 2016, al finalizar el proceso completo de intervención. Algunas de estas familias venían siendo atendidas en el período anterior (año académico 2015) y, por ello, hacen referencia a las modificaciones del modelo al integrar a las visitas domiciliarias ya instaladas, el trabajo del equipo profesional en el centro, la intervención en duplas y los talleres interactivos triangulares (Andreucci-Annunziata \& Guajardo, 2016). 


\section{Sobre la modalidad general de trabajo en el centro AT}

Las familias sostienen que la «nueva» modalidad va acorde a los requerimientos de sus hijos o hijas, según la etapa en la que se encuentran. Además, plantean que se sienten apoyadas y orientadas en los espacios generados por los distintos profesionales, puesto que se entregan herramientas útiles y se produce un diálogo constante.

En comparación al año pasado, este año yo sentí un complemento, en cierta manera, (a mi hijo), a mí, a mi mamá que es la persona que me ayuda a criar (a mi hijo), de partida por la psicóloga, para mí en lo personal fue maravilloso, ya después que tuviera fonoaudióloga y que le ayuda con el trastorno de deglución, la educadora diferencial o la terapeuta ocupacional, para mi esas cosas fue un mayor complemento que el año pasado, o sea todos estaban pendiente de [él], las tías por el whatsapp me daban tips o yo les preguntaba. A diferencia del año pasado, este año sentí que fue más apoyado, en cierta manera, con todos los profesionales que se pusieron a disposición de nosotros. (TF, Fam. 28, sábado oz de diciembre de 2016)

Nosotros no tenemos mucho punto de comparación porque somos nuevos, para mi ideal que sea en la casa porque al niño le sirve que lo vean en su entorno diario, ese mix de la casa y además acá yo lo encuentro genial. (MS, Fam. 3o, sábado 10 de diciembre de 2016)

(...) va a depender de la etapa en que estamos con los hijos; en una primera etapa que también yo estuve en la estimulación temprana en otra universidad, también las chicas iban a la casa, y creo que en esa etapa cuando son chiquititos que tienen meses, menos de un año, es súper importante el apoyo que tienen porque nos enseñan y además tu no siempre tienes tiempo para salir a las terapias y vienen niñas y te enseñan y uno puede aplicar. Pero en la etapa que está mi [hija] que está más grande ha sido genial el cambio porque además hemos estado súper respaldados por la fonoaudióloga y los talleres del día sábado, lo encuentro genial de poder compartir con chicos igual que ella, con sus pares, porque en el jardín ella es la única con Síndrome de Down, entonces está toda la semana en otra y los sábados está especialmente con sus pares que también es importante en la vida de ellos. (EH, Fam. 23, sábado o3 de diciembre de 2016)

CET es como fantástico llegar, que acá no hay fines de lucro, que acá todos estamos en la misma, yo creo que el centro da una ventanita a que uno vaya proyectándose con los niños. Los chiquillos aprenden, nosotros aprendemos mucho más. Yo creo que a los que estamos aquí nos pasa lo mismo. (JY, Fam. 24, sábado 10 de diciembre de 2016) 


\section{Sobre el trabajo en duplas profesionales}

En términos generales, se visualiza que el trabajo realizado por el equipo es fundamental, puesto que se involucra con los niños y las niñas y sus familias. Se resalta el nivel que posee el equipo en términos profesionales y personales.

Hay que agradecer el nivel de profesionalismo de las duplas que han acompañado a los niños; nosotros hemos recibido un trato, un acompañamiento, una orientación súper buena, no tenemos en si una comparación con centros de mayor nivel socioeconómico derechamente, pero si tenemos la comparación de poder ver cómo los chicos se involucran en esto y creo que eso es una de las principales cosas y muy importantes a la hora de estimular a un pequeño con esa condición, entonces nosotros lo hemos notado. (EF, Fam. 12, sábado 10 de diciembre de 2016)

La calidad de las duplas profesionales que hay, aparte de ser buenos profesionales tienen calidad humana que no muchos profesionales lo tienen, a mí me ha tocado que en otras partes voy, le hacen la terapia y eso sería, acá tienen la chispa para escucharte, para guiarte. (CH, Fam. 20, sábado 10 de diciembre de 2016)

\section{Sobre el programa de acompañamiento a padres, madres o cuidadores}

Finalmente, respecto de la generación del espacio denominado Taller para padres, madres o cuidadores se le entrega un sentido especial, puesto que abre la posibilidad del diálogo, el compartir con otros padres y madres, trabajar en aquellos aspectos que necesitan resolver en su función de progenitores. Este espacio semanal se visualiza de manera positiva posibilitando la participación de las familias como protagonistas de sus propias inquietudes, soluciones intentadas y temores. Esto, entendiendo que el cuidado como fenómeno social, involucra a distintos individuos y, por tanto, allí radica la importancia de la generación y ampliación de redes de apoyo (Moreno \& Granada, 2014).

Y este año cambió totalmente el sistema y este año, comparto con los papás, que nos incluyen a nosotros, en el sentido de venir acá y que los chiquillos vean sus capacidades. Y aparte mientras que ellas están es eso, nosotros poder estar aquí como papás, poder conversarlo, nos vamos dando tips entre nosotros mismos en cosas que yo decir «pucha» pero como voy a lograr que [mi hija] haga esto. Entonces, como que eso te ayuda porque no es netamente que los niños estén trabajando en eso porque hoy día avanzo en algo, no, estamos avanzando con los papás y los niños con sus pares. Entonces yo siento que los niños por lo menos este año ha sido bueno el cambio y eso de que ahora trabajen con nosotros dan las ganas de seguir (CV, Fam. 22, sábado 10 de diciembre de 2016) 
En relación con lo anterior, las familias agregan que es posible visualizar cómo se ha ido desarrollando el centro, la importancia que tiene este proyecto, en el sentido de que se genera un espacio, un lugar real y «simbólico» en el cual se trabaja tanto con los niños y las niñas como con sus familias.

Estaba sorprendida la doctora del nivel de desarrollo que ha alcanzado el centro y aplaudía el hecho de que la universidad haga suyo este proyecto y lo pueda seguir expandiendo. (EH, Fam. 23, sábado o3 de diciembre de 2016).

Bueno ese es nuestro resumen y estamos súper contentos porque hemos visto evolución y apoyo, porque a veces ni siquiera es el desarrollo del niño sino que también el mío como papá, porque en estas conversaciones te estimulan, te guían, entonces el mismo avance que puede tener nuestro hijo puede ser de cualquiera de ustedes, que a veces, sobre todo al principio, es súper complejo porque es un mundo totalmente nuevo, desconocido, entonces uno no sabe qué hacer, cuando uno se da cuenta que también hay gente que va caminando en la misma senda, cambia la cosa, así que agradecido. (JY, Fam. 24, sábado 10 de diciembre de 2016)

\section{Conclusiones}

En general, se visualiza que tanto el diseño como la implementación del modelo van bien encaminados en la percepción, resultados obtenidos y expectativas de atención y desarrollo de las propias familias. En las limitaciones, lo que queda por avanzar y mejorar, las madres y los padres plantean sus requerimientos asociados a una mayor participación tanto en la toma de decisiones como en la retroalimentación de los avances de sus hijos e hijas. En lo específico:

Entonces yo creo que debería ir él, informarse más, en cada sesión decir esto se hizo, en esto se avanzó o en esto quedó pendiente. (EF, Fam. 22, sábado 1o de diciembre de 2016)

Cuando los niños salen en ese momento deberían hacer una pequeña charla de unos minutos, para qué tan personalizado tampoco, ya nos conocemos todos, los niños comparten. Pero que ellos se den el tiempo de hablarnos a nosotros como papás de los avances y en los no avances de los niños». (CH, Fam. 2, sábado 10 de diciembre de 2016)

Por tanto, avanzar hacia un enfoque centrado en la familia supone asumir los desafíos, retos y oportunidades de una paulatina mayor involucramiento de padres, madres o 
cuidadores con sus respectivas y legítimas demandas al dispositivo de AT implementado, a sus profesionales y, especialmente, a su activa participación en los avances de aprendizaje-desarrollo de sus hijos e hijas.

Como se ha constatado, tanto en la revisión de la literatura como en este reporte particular, los programas de AT que incluyen a las familias en su diseño e implementación conllevan importantes beneficios a sus hijos e hijas, evidenciándose los efectos tanto a corto como a mediano y largo plazo. Los efectos en los niños y niñas pueden sintetizarse en dos: 1) mejora en la salud, desarrollo físico y psicomotor; y 2) logros cognitivos, socioemocionales y educacionales. Estos efectos no fueron recogidos en esta comunicación. Los efectos en las figuras significativas, madres, padres o cuidadores (foco de este trabajo) remiten a: 1) mejorías en la relación padre-hijo, en la sensibilidad y competencia parental; y 2) disminución en problemáticas de salud mental (Guralnick, 2016; McWilliam, 2016). Se agregan, de acuerdo a lo reportado por los propios padres y madres de este estudio, un mayor protagonismo en la toma de decisiones e involucramiento paulatino en el avance del desarrollo y aprendizaje de sus hijos e hijas.

Sin duda, la familia requiere constituirse en el eje central o foco de la atención y, en consideración a la edad de los niños y niñas, internalizar que las actuaciones tempranas deben comenzar desde el principio de la vida de sus hijos e hijas e incluso antes en la gestación. Esto, considerando que el modelo transaccional del cuidado, implica concebir las interacciones vinculares entre niños y niñas y un/a sujeto cuidador/a que contribuye en el estado de desarrollo (Moreno \& Granada, 2014). Por otro lado, aporta al bienestar subjetivo y al protagonismo de madres, padres o figuras relevantes-significativas de niños y niñas con condición de SD en su rol de cuidadores/as posibilita lograr los propósitos planteados en las políticas públicas tanto a nivel latinoamericano como mundial (Acosta \& Reyes, 2017; Irarrázaval, Prieto, \& Armijo, 2016) y en el espíritu de los equipos profesionales que requieren ceder terreno a la opinión y planteamientos cada vez más informados de padres, madres y familiares. De este modo, se afianza el trabajo sistémico colaborativo del equipo a través de las visitas domiciliarias, del trabajo en duplas y del trabajo conjunto con los padres y madres en grupos triangulares en el centro de estimulación (CET) y atención tempranas.

Sin duda, implementar una opción modélica como la propuesta supone considerar la AT como un derecho del niño y niña, controlando rigurosamente la ecología socioafectiva en la perspectiva de una educación inclusiva en primera infancia. Lo anterior, ubica a los padres y madres como protagonistas de los cuidados cotidianos, aportándoles 
conocimiento del neurodesarrollo de sus hijos e hijas y, a su vez, de la dinámica, sistemas de apego, de crianza, de cuidado y entorno familiar (Flores \& Porges, 2017; González et al., 2017; Palacios-Hernández, 2016; Viloria \& González, 2017). Se requiere monitorear, al mismo tiempo, las indicaciones de seguimiento del equipo multiprofesional emitidas en los informes exhaustivos de egreso del sistema ya que tienden a retornar, eventualmente, las frustraciones, angustias e inseguridades en el rol y funciones parentales. Los centros AT pueden prevenir la iniciación tardía de tratamientos, actuando acorde a las necesidades del niño, niña y su familia, depositando confianza en los reportes, sensibilidad, inquietudes y cuestionamientos de padres, madres y cuidadores. De este modo, se le devuelve a la familia el protagonismo en su rol y funciones sin dejar de acompañarla y supervisarla a lo largo de todo el proceso, incluso en el ingreso al jardín de infantes, materia de una próxima publicación dada su relevancia en la transición educativa verdaderamente inclusiva.

Como temáticas emergentes y limitantes se necesita reparar en la calidad de los servicios de AT y sus requisitos formativos. El contar con centros que articulan disciplinas al interior de instituciones universitarias o de formación superior (terciaria), ofrece ciertas garantías de actualización permanente e investigación sistemática; no obstante, se requiere de una validación constante del modelo por sobre la propuesta médica más conocida y legitimada socialmente. La investigación en este ámbito es relevante, pero también lo es la percepción de los y las afectadas y, a su vez, profundamente invisibilizados/as por perspectivas centradas en el déficit y el que la «padece».

La evaluación de los efectos de la implementación de dispositivos de AT en los niños, niñas y sus familias permitirá atender más efectivamente a la formación de expertos y expertas en diversas temáticas, tales como: niños y niñas con trastornos del espectro autista; niños y niñas con problemas de comportamiento; niños y niñas abandonados (de padres con problemas de salud mental, por ejemplo); niños y niñas de familias desfavorecidas o vulnerables socioeconómicamente; niños y niñas prematuros(as), niños y niñas inmigrantes, entre los más relevantes. Tanto la particularidad e intensidad de las intervenciones diseñadas e implementadas como su permanencia en el tiempo se constituyen en elementos claves de éxito; en ello, resulta esencial la activa participación de las familias (padres y madres, hermanos y hermanas, tíos y tías, abuelos y abuelas).

Ahora bien, una de las dimensiones limitantes del estudio que, debido a los tiempos, no fue posible observar y analizar, se refiere a la participación del equipo multidisciplinario en esta perspectiva sistémica; esto considerando que debido al diseño de las inter- 
venciones pasan a ser actores significativos y, de una u otra forma, son quienes también componen y aceitan la red de cuidado familiar-habitual. En esta línea, es necesario abordar la noción de cuidado como práctica que ha sido invisibilizada desde la institucionalidad y, que en mayor o menor grado, contribuye a la desigualdad, particularmente en el caso de familias con niños y niñas con SD, lugar donde la culpa y la maternalización apuntan a asignar a la madre/mujer dicha responsabilidad. Este aspecto será abordado en una próxima comunicación.

\section{Referencias}

Acosta, J. P. P., \& Reyes, M. M. (2017). Sistemas educativos: un análisis documental «programas de atención integral a la primera infancia de Colombia y México». Jóvenes en la ciencia, 2(1), 1736-1740.

Álvarez, M. I. C., Alonso, M. A. V., \& Amor, A. M. (2016). La participación familiar es un requisito imprescindible para una escuela inclusiva. Revista Latinoamericana de Educación Inclusiva, 10(1), 99-113. https://doi.org/10.4067/so718-73782016000100006

Andreucci-Annunziata, P. (2016, noviembre). Educación inclusiva en Chile: la experiencia de CET-Ucinf. Ponencia, III Jornadas Nacionales y I Jornadas Internacionales sobre «Familia y atención a la diversidad desde una perspectiva ecológica». Centro de Formación del Profesorado, Universidad Complutense de Madrid, Madrid, España.

Andreucci-Annunziata, P., \& Guajardo, G. (2016). El potencial creativo de los grupos triangulares: una aproximación intersubjetiva y dialógica. Akademeia, 15(2), 65-76.

Ancheta, A. (2018). Equidad y educación de la primera infancia en la agenda educativa mundial. Revista Latinoamericana de Ciencias Sociales, Niñez y Juventud, 17(1), 47-59.

Booth, T., \& Ainscow, M. (2015). Guía para la educación inclusiva: desarrollando el aprendizaje y la participación en los centros escolares. Madrid: Fuhem y OEI. Recuperado de: https://downgalicia.org/wp-content/uploads/2018/o1/Guia-para-la-Educacion-Inclusiva.pdf

Cara, M., Sola, T., Aznar, I., \& Fernández, F. (2015). Análisis organizativo de las clases de inclusión escolar en Francia. Revista Latinoamericana de Ciencias Sociales, Niñez y Juventud, 13(2), 795-807. https://doi.org/10.1160o/1692715x.13217200314

Esquivel, M. (2015). Children with Down Syndrome: Life stories of parents. Revista Electrónica Educare, 19(1), 311-331. 
Federación Estatal de Asociaciones de Profesionales de Atención Temprana. (2000). Libro blanco de la atención temprana. Madrid: Real Patronato sobre Discapacidad.

Flores, P. J., \& Porges, S. W. (2017). Group psychotherapy as a neural exercise: Bridging Polyvagal Theory and Attachment Theory. International Journal of Group Psychotherapy, 26, 1-21. https://doi.org/10.1080/00207284.2016.1263544

García, F. A., Escorcia, C. T., Sánchez, M. C., Orcajada, N., \& Hernández, E. (2014). Atención temprana centrada en la familia. Siglo Cero. Revista Española sobre Discapacidad Intelectual, 45(3), 6-27.

González, R. A. M., Blanco, L. A., \& Herrero, M. D. H. P. (2017). La percepción de padres y madres en el ejercicio del rol parental. Revista Infad de Psicología., 6(1), 47-56. https://doi.org/10.17060/ijodaep.2014.n1.v6.716

Guralnick, M. J. (2011). Why early intervention works: A systems perspective. Infants and Young Children, 24(1), 6. https://doi.org/10.1097/iyc.obo13e3182002cfe

Guralnick, M. J. (2016). Early intervention: The integration of science and practice. Journal of Intellectual Disability Research, 60(7), 624-635.

Irarrázaval, M., Prieto, F., \& Armijo, J. (2016). Prevención e intervenciones tempranas en salud mental: una perspectiva internacional. Acta Bioethica, 22(1), 37-5o. http://dx.doi.org/ $10.4067 / S_{1726-569 X 2016000100005}$

Kosubik, M., Síthova, S., Kajanová, A., \& Rác, I. (2018). Is Feuerstein's instrumental enrichment (FIE) a good method for social inclusion of poor Slovak children in school? A study focused on social and educational levels. https://doi.org/10.1016/j.kontakt.2017.10.006

Lozano-Vicente, A. (2016). Los derechos del niño: cuestiones sobre su fundamentación. Revista Latinoamericana de Ciencias Sociales, Niñez y Juventud, 14(1), 67-79. https://doi.org/10.1160o/1692715x.1417090915

McWilliam, R. (2016). Metanoia en atención temprana: transformación a un enfoque centrado en la familia. Revista Latinoamericana de Inclusión Educativa, 10(1), 133-153. http://dx.doi.org/10.4067/So718-73782016000100008

Morales-Cabello, C., \& Andreucci-Annunziata, P., Eisendecher, E. (2018, noviembre). Cuidados y vínculo, desde la perspectiva de género y discapacidad: análisis de la experiencia. Comunicación. Primer Congreso Latinoamericano de Estudios de Género y Cuidados. Departamento de Sociología, Facultad de Ciencias Sociales, Universidad de La República, Montevideo, Uruguay.

Moreno, M., \& Granada, P. (2014). Interacciones vinculares en el sistema de cuidado infantil. Revista Latinoamericana de Ciencias Sociales, Niñez y Juventud, 12(1), 121-139. https://doi.org/10.11600/1692715x.1216052513 
Palacios-Hernández, B. (2016). Alteraciones en el vínculo materno-infantil: prevalencia, factores de riesgo, criterios diagnósticos y estrategias de evaluación. Revista de la Universidad Industrial de Santander. Salud, 48(2), 164-176. https://doi.org/10.18273/revsal.v48n2-2016001

Palacios, A., \& Bariffi, F. J. (2014). La discapacidad como una cuestión de derechos humanos: una aproximación a la Convención Internacional sobre los Derechos de las Personas con Discapacidad. Madrid: Ediciones Cinca.

Palomares, A., Sánchez, B., \& Garrote, D. (2016). Educación inclusiva en contextos inéditos: la implementación de la Pedagogía Hospitalaria. Revista Latinoamericana de Ciencias Sociales, Niñez y Juventud, 14(2), 1507-1522. https://doi.org/10.1160o/1692715x. 14242240815

Pardo, M., Gómez, M., \& Edwards, M. (2012). Test de aprendizaje y desarrollo infantil (Tadi). Santiago de Chile: Ciae, Cide.

Rockel, J., \& Sansom, A. (2015). The language of the body: Body wisdom in the first years. The first years Ngā Tau Tuatahi. New Zealand Journal of Infant and Toddler Education, $17(1), 11-16$.

Rodríguez, H., Jiménez, M., \& Sánchez, S. (2018). Construcción del discurso en torno a la educación inclusiva. Revista Electrónica Interuniversitaria de Formación del Profesorado, 21(1), 185-217. https://doi.org/10.6018/reifop.21.1.305771

Shulman, C. (2016). Theoretical bases of intervention in infant and early childhood mental health. Berna: Springer.

Simón, C., Giné, C., \& Echeita, G. (2016). Escuela, familia y comunidad: construyendo alianzas para promover la inclusión. Revista Latinoamericana de Inclusión Educativa, 10(1), 25-42. http://dx.doi.org/10.4067/So718-73782016000100003

Stake, R. E. (2010). Investigación con estudio de casos. Madrid: Morata.

Viloria, C. A., \& González, A. F. (2017). Las prácticas de crianza de los padres: su influencia en las nuevas problemáticas en la primera infancia. Revista de Educación Inclusiva, $9(1), 30-42$. 